\title{
Predictive factors for hyperprogressive disease during nivolumab as anti-PD1 treatment in patients with advanced gastric cancer
}

\author{
Akinori Sasaki $^{1,2}$. Yoshiaki Nakamura ${ }^{1}$. Saori Mishima ${ }^{1} \cdot$ Akihito Kawazoe $^{1}$ - Yasutoshi Kuboki ${ }^{1} \cdot$ Hideaki Bando $^{1}$. \\ Takashi Kojima ${ }^{1} \cdot$ Toshihiko Doi $^{1}$ - Atsushi Ohtsu ${ }^{1}$ - Takayuki Yoshino ${ }^{1} \cdot$ Takeshi Kuwata $^{3}$ - Tetsuo Akimoto ${ }^{2,4}$. \\ Kohei Shitara ${ }^{1}$
}

Received: 1 November 2018 / Accepted: 25 December 2018 / Published online: 9 January 2019

(c) The International Gastric Cancer Association and The Japanese Gastric Cancer Association 2019

\begin{abstract}
Background Hyperprogressive disease (HPD) during treatment with anti-programmed death-1/programmed death-ligand 1 monoclonal antibodies has anecdotally been reported in some types of cancers, but is not well-characterized in patients with advanced gastric cancer (AGC).

Methods Total 62 AGC patients treated with nivolumab in a single institution from September 2017 to April 2018 were enrolled in this study. Tumor responses were assessed according to Response Evaluation Criteria in Solid Tumors version 1.1 , and HPD was defined as $\geq$ two fold increase in tumor growth rate. Clinicopathological and molecular characteristics associated with HPD were also investigated.

Results Thirteen of 62 patients (21\%) developed HPD after nivolumab treatment. Overall survival (OS) and progression-free survival (PFS) were significantly shorter in patients with HPD than in patients without HPD (median OS: 2.3 months vs. not reached, $P<0.001$; median PFS: 0.7 months vs. 2.4 months, $P<0.001$ ). Liver metastases (77\% vs. 41\%), Eastern Cooperative Oncology Group (ECOG) performance status (PS) of 1 or 2 (77\% vs. 29\%), and a large sum of target lesion diameters at baseline (median $104.2 \mathrm{~mm}$ vs. $44.9 \mathrm{~mm}$ ) were significantly associated with HPD. Absolute neutrophil count (ANC) and C-reactive protein (CRP) level significantly increased in the first 4 weeks in only patients with HPD.

Conclusions HPD was observed in AGC patients treated with nivolumab and correlated with some clinicopathological characteristics. Elevations in ANC and CRP levels upon treatment might indicate HPD.
\end{abstract}

Keywords Nivolumab · PD-1 inhibitor · Gastric cancer · Hyperprogressive disease

Abbreviations
$\begin{array}{ll}\text { PD-1 } & \text { Anti-programmed death-1 } \\ \text { PD-L1 } & \text { Programmed death-ligand 1 } \\ \text { mAbs } & \text { Monoclonal antibodies } \\ \text { HPD } & \text { Hyperprogressive disease } \\ \text { AGC } & \text { Advanced gastric cancer } \\ \text { OS } & \text { Overall survival } \\ \text { PFS } & \text { Progression-free survival }\end{array}$

Akinori Sasaki and Yoshiaki Nakamura contributed equally to this work.

Electronic supplementary material The online version of this article (https://doi.org/10.1007/s10120-018-00922-8) contains supplementary material, which is available to authorized users.

Kohei Shitara

kshitara@east.ncc.go.jp

Extended author information available on the last page of the article
ECOG PS Eastern Cooperative Oncology Group performance status

ANC Absolute neutrophil count

CRP C-reactive protein

HER2 Human epidermal growth factor receptor 2

MMR Mismatch repair

EBV Epstein-Barr virus

IHC Immunohistochemistry

FISH Fluorescence in situ hybridization

TC Tumor cell

IC Immune cell

CPS Combined positive score

MLH1 Anti-mutL homolog 1

MSH2 Anti-mutS homolog 2

PMS2 Anti-postmeiotic segregation increased 2

MSH6 Anti-mutS homolog 6

EBER EBV-encoded RNA

TMB Tumor mutation burden 


$\begin{array}{ll}\text { mt/MB } & \text { Mutations/megabase } \\ \text { NLR } & \text { Neutrophil-to-lymphocyte ratio } \\ \text { LDH } & \text { Lactate dehydrogenase } \\ \text { TGK } & \text { Tumor growth kinetics } \\ \text { TGK }_{R} & \text { TGK ratio } \\ \text { HR } & \text { Hazard ratio } \\ \text { CI } & \text { Confidence interval } \\ \text { PR } & \text { Partial response } \\ \text { SD } & \text { Stable disease } \\ \text { PD } & \text { Progressive disease } \\ \text { MAPK } & \text { Mitogen-activated protein kinase } \\ \text { MDSC } & \text { Myeloid-derived suppressor cell }\end{array}$

\section{Introduction}

Gastric cancer (GC) is the fifth most common cancer and the third leading cause of cancer mortality worldwide [1]. Although some chemotherapy regimens, including a platinum and fluoropyrimidine combination, trastuzumab (for HER2-positive cases), taxanes, irinotecan, and ramucirumab have been shown to improve the survival outcomes of patients with advanced GC (AGC) [2-6], the prognosis remains poor, with the median survival being approximately 1 year. Therefore, further therapeutic development is needed for AGC.

Nivolumab, a fully human IgG4 monoclonal antibody $(\mathrm{mAb})$ against programmed death-1 (PD-1), is an immune checkpoint inhibitor that enhances antitumor T-cell activity through the inhibition of immune checkpoints. It has been shown to have efficacy against various types of malignancies [7-11]. A recent randomized phase III trial, ATTRAC TION-2 (ONO-4538-12), demonstrated that nivolumab treatment after two or more previous chemotherapy regimens in AGC patients had a survival benefit compared to that associated with placebo [12]. Pembrolizumab, another PD-1 mAb, also demonstrated encouraging antitumor activity with acceptable safety for programmed death-ligand 1 (PD-L1)-positive AGC in phase II and III trials [13, 14].

Recently, anti-PD-1/PD-L1 mAbs have anecdotally been reported to cause rapid progression of some types of cancers, which is called hyperprogressive disease (HPD) [15-18]. To avoid the potential harm, it is necessary to identify clinical or molecular factors for predicting HPD. However, the characteristics of HPD in AGC patients treated with anti-PD-1/ PD-L-1 mAbs remain unclear. Therefore, in this study, we assessed HPD by estimating tumor growth kinetics during nivolumab treatment in AGC and evaluated the clinicopathological and molecular factors associated with HPD.

\section{Methods}

\section{Patients}

A retrospective study was performed to evaluate HPD in patients with AGC who received nivolumab from September 2017 to April 2018 at the National Cancer Center Hospital East. Patients who met the following criteria were included: (1) presence of histologically proven gastric adenocarcinoma; (2) receipt of treatment with two or more previous chemotherapy regimens; (3) underwent nivolumab treatment; (4) an Eastern Cooperative Oncology Group performance status (ECOG PS) of 0 to 2; and (5) availability of a computed tomography (CT) scan during previous chemotherapy (pre-baseline CT), before initiation of nivolumab treatment (baseline CT), and within 3 months after the initiation of nivolumab treatment (post-CT). Tumor responses were assessed according to Response Evaluation Criteria in Solid Tumors version 1.1 (RECIST v1.1) [19]. Patients who did not have any measurable lesions, as judged based on RECIST v1.1, were excluded. All CT scans were independently reviewed by two or more physicians (A. S., Y. N., and K. S.). All patients provided written, informed consent prior to participating in this observational study. The study protocol was approved by the Institutional Review Board at the National Cancer Center.

\section{Molecular characteristics}

Molecular characteristics, such as the status of the human epidermal growth factor receptor 2 (HER2), PD-L1, mismatch repair (MMR), Epstein-Barr virus (EBV), and genomic alterations, were analyzed with formalin-fixed paraffin-embedded tissue specimens from archival tissue samples if available. Immunohistochemistry (IHC) using a monoclonal anti-HER2 antibody (PATHWAY HER2 [4B5], Ventana, Tucson, AZ) and fluorescence in situ hybridization (FISH) using the PathVysion HER-2 probe kit (Abbott Laboratories, Abbott Park, IL, USA) were performed to assess HER2 status, and HER2 positivity was defined as IHC $3+$ or IHC $2+$ and FISH positive. IHC for PD-L1 was performed using an anti-PD-L1 rabbit monoclonal antibody [VENTANA PD-L1 (SP142) Assay, Ventana], and PD-L1 positivity on tumor cells (TCs) or immune cell (ICs) was defined as the presence of $\geq 1 \%$ of TCs or ICs with membrane staining. The combined positive score (CPS), which was the number of cells showing PD-L1 staining (TCs, lymphocytes, and macrophages) divided by the total number of viable TCs multiplied by 100 , was also determined. MMR status was assessed by 
IHC using monoclonal anti-mutL homolog 1 (MLH1, ES05), anti-mutS homolog 2 (MSH2, FE11), anti-postmeiotic segregation increased 2 (PMS2, EP51), and anti-mutS homolog 6 antibodies (MSH6, EP49) (Agilent Technologies, Santa Clara, CA), and tumors lacking MLH1, MSH2, PMS2, or MSH6 expression were considered as MMR deficient (D-MMR). Chromogenic in situ hybridization for EBV-encoded RNA (EBER) using fluorescein-labeled oligonucleotide probes (INFORM EBER Probe, Ventana) was performed to assess EBV status [20]. Genomic alterations were assessed using Oncomine ${ }^{\mathrm{TM}}$ Comprehensive Assay version 3 or Oncomine ${ }^{\mathrm{TM}}$ Cancer Research Panel (Thermo Fisher Scientific, Waltham, MA), which allows detection of gene mutations, copy number variants, and fusions across multiple genes (Additional File 1: Table S1). Tumor mutation burden (TMB) was defined as the number of non-synonymous mutations, including indel, per megabase $(\mathrm{mt} / \mathrm{Mb})$ of genome in tumor tissue. Known germline variants in dbSNP and East Asian population of 1000 Genomes or ExAC database were not counted.

\section{Laboratory data}

To determine whether serial monitoring of laboratory data can predict HPD, the following laboratory data at the beginning of nivolumab treatment and at the first 2 and 4 weeks of therapy were collected: carcinoembryonic antigen (CEA), carbohydrate antigen (CA)19-9, C-reactive protein (CRP), absolute neutrophil count (ANC), lymphocyte count, neutrophil-to-lymphocyte ratio (NLR), lactate dehydrogenase (LDH) level, and albumin level.

\section{Definition of HPD}

The time of pre-baseline, baseline, and post-CT scanning was defined as $T_{\mathrm{PRE}}, T_{0}$, and $T_{\mathrm{POST}}$, respectively. The sum of the largest diameters of the target lesions according to RECIST v1.1 at pre-baseline, baseline, and post-CT was defined as $S_{\mathrm{PRE}}, S_{0}$, and $S_{\mathrm{POST}}$, respectively [16].

Tumor growth kinetics (TGK) were assessed as described previously [16]. Briefly, $\mathrm{TGK}_{\mathrm{PRE}}$ was calculated as the difference of the sum of the largest diameters of the target lesions per unit of time between pre-baseline and baseline imaging: $\left(S_{0}-S_{\mathrm{PRE}}\right) /\left(T_{0}-T_{\mathrm{PRE}}\right)$. Similarly, $\mathrm{TGK}_{\mathrm{POST}}$ was calculated as $\left(S_{\mathrm{POST}}-S_{0}\right) /\left(T_{\mathrm{POST}}-T_{0}\right)$. We defined $\mathrm{TGK}_{\mathrm{POST}} / \mathrm{TGK}_{\mathrm{PRE}}$ as TGK ratio $\left(\mathrm{TGK}_{\mathrm{R}}\right)$. According to the previous studies [15-17], HPD was defined as $\mathrm{TGK}_{\mathrm{R}} \geq 2$ and $>50 \%$ increase in tumor burden compared to that at pre-treatment imaging.

\section{Statistical analyses}

Statistical comparisons of baseline characteristics between HPD and non-HPD patients were performed using $\chi^{2}$ or Fisher's exact test for categorical data and Student's test or Mann-Whitney's test for continuous variables. Progressionfree survival (PFS) was estimated from the date of initiation of nivolumab treatment to the date of disease progression or death from any cause. Overall survival (OS) was defined as the interval between the date of initiation of nivolumab treatment and the date of mortality due to any cause. PFS and OS were determined and presented graphically using the Kaplan-Meier method. Survival rates at various times and $95 \%$ confidence intervals were also determined. The survival curves were compared using the long-rank test and hazard ratio (HR) and 95\% confidence interval (CI) was determined using the Cox's proportional-hazards model for the comparison of the HPD and non-HPD groups. Multivariate analysis was conducted to adjust HR using clinical factors which showed difference between HPD and non-HPD groups. All statistical analyses were performed with $5 \%$ alpha risk or 95\% confidence interval using SPSS version 25 (IBM, Chicago, IL, USA).

\section{Results}

\section{Patients' characteristics}

Among 73 AGC patients treated with nivolumab between September 2017 and April 2018, 11 were excluded because of the absence of post-CT imaging (four patients) or the absence of measurable disease on CT imaging (seven patients). Thus, in total, 62 patients were eligible for inclusion in this study (Fig. 1).

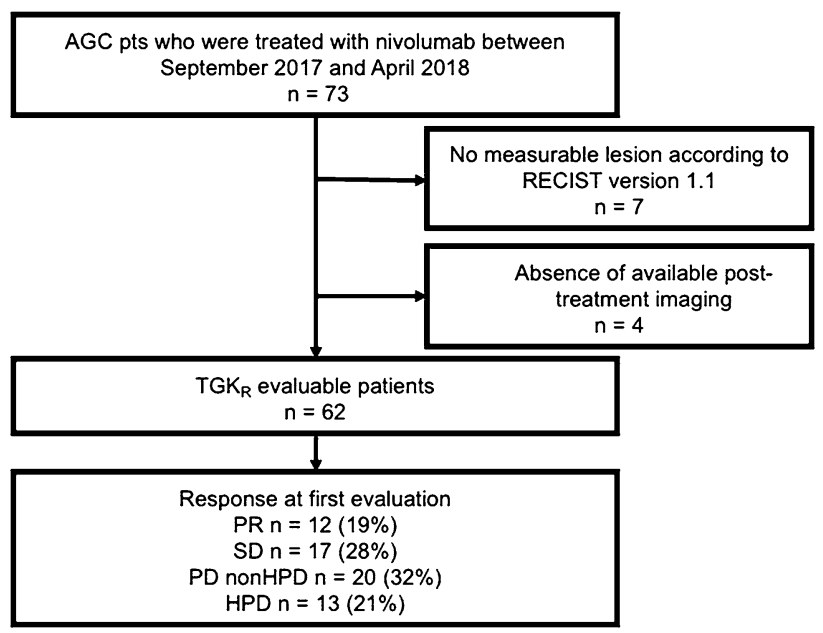

Fig. 1 Consort flow diagram 
Table 1 Patients' characteristics

\begin{tabular}{|c|c|c|c|c|c|}
\hline Features & Non-HPD $(n=49)$ & $\operatorname{HPD}(n=13)$ & HPD ratio $(\%)$ & OR & $P$ value \\
\hline Age, $\geq 65, n(\%)$ & $31(63.3)$ & $9(69.2)$ & 22.5 & 1.3 & 0.76 \\
\hline Male, $n(\%)$ & $33(71.4)$ & $12(92.3)$ & 26.7 & 4.8 & 0.16 \\
\hline \multicolumn{6}{|l|}{ ECOG PS, $n(\%)$} \\
\hline 0 & $35(71.4)$ & $3(23.1)$ & 7.9 & Ref. & \multirow[t]{2}{*}{0.003} \\
\hline 1 or 2 & $14(28.6)$ & $10(76.9)$ & 41.7 & 8.3 & \\
\hline \multicolumn{6}{|l|}{ Histology, $n(\%)$} \\
\hline Intestinal & $24(49.0)$ & $6(46.2)$ & 20 & Ref. & \multirow[t]{2}{*}{0.81} \\
\hline Diffuse & $25(51.0)$ & $7(53.8)$ & 21.9 & 1.1 & \\
\hline \multicolumn{6}{|c|}{ Previous treatment regimens, $n(\%)$} \\
\hline 2 & $24(49.0)$ & $4(30.8)$ & 14.3 & Ref. & \multirow[t]{2}{*}{0.35} \\
\hline$\geq 3$ & $25(51.0)$ & $9(69.2)$ & 26.5 & 2.2 & \\
\hline \multicolumn{6}{|l|}{ Previous gastrectomy, $n(\%)$} \\
\hline No & $28(57.1)$ & $10(76.9)$ & 26.3 & Ref. & \multirow[t]{2}{*}{0.22} \\
\hline Yes & $21(42.9)$ & $3(23.1)$ & 12.5 & 0.4 & \\
\hline \multicolumn{6}{|c|}{ Previous radiation therapy, $n(\%)$} \\
\hline No & $46(93.9)$ & $12(92.3)$ & 20.7 & Ref. & \multirow[t]{2}{*}{1.00} \\
\hline Yes & $3(6.1)$ & $1(7.7)$ & 25 & 1.3 & \\
\hline \multicolumn{6}{|c|}{ Organs with metastases, $n(\%)$} \\
\hline$\leq 2$ & $35(71.4)$ & $6(46.2)$ & 14.6 & Ref. & \multirow[t]{2}{*}{0.19} \\
\hline$\geq 3$ & $14(28.6)$ & $7(53.8)$ & 33.3 & 2.9 & \\
\hline \multicolumn{6}{|l|}{ Site of metastases, $n(\%)$} \\
\hline Liver & $20(40.8)$ & $10(76.9)$ & 33.3 & 4.8 & 0.029 \\
\hline Lung & $9(18.4)$ & $2(15.4)$ & 18.2 & 0.81 & 1.00 \\
\hline Peritoneum & $25(51.0)$ & $6(46.2)$ & 19.4 & 0.82 & 1.00 \\
\hline Lymph node & $42(85.7)$ & $11(84.6)$ & 20.8 & 0.92 & 1.00 \\
\hline Other & $14(28.6)$ & $4(30.8)$ & 22.2 & 1.1 & 1.00 \\
\hline$S_{0}(\mathrm{~mm}), \geq$ median, $n(\%)$ & $20(40.8)$ & $11(84.6)$ & 35.5 & 8.0 & 0.003 \\
\hline
\end{tabular}

$H P D$ hyperprogressive disease, $O R$ odds ratio, ECOG PS Eastern Cooperative Oncology Group performance status, ref reference, $S_{0}$ the sum of the largest diameters of target lesions estimated according to RECIST v1.1 at baseline

Table 1 shows patient characteristics. The median patient age was 67 (range 25-86) years, and 47 patients (76\%) were male. Thirty-eight patients (61\%) had an ECOG PS of 0, whereas the remaining 24 patients (39\%) had a PS of 1 or 2 at the initiation of nivolumab treatment. Thirty-four patients $(56 \%)$ had been treated with three or more lines of the previous chemotherapies before nivolumab treatment. The most common metastatic site was the lymph node (85\%), followed by the liver and peritoneum. The median $S_{0}$ (the sum of the diameters of the target lesion at baseline) was 59.4 (range 16.0-260.9) $\mathrm{mm}$.

Data on HER2, PD-L1, MMR, EBV, and genomic alteration statuses were available in $61,53,55,57$, and 47 patients. Fourteen patients showed HER2-positive tumors. PD-L1 expression on TCs or ICs was identified in 47 patients. Fourteen patients $(26.4 \%)$ showed tumors with a PD-L1 CPS of 10 or higher. Eight patients (14.5\%) were found to have a D-MMR status, and four patients showed EBV-positive tumors $(7.1 \%)$.

\section{Response to treatment}

The median follow-up time was 8.1 (range 5.9-10.0) months. The best overall responses were partial response (PR) in 12 patients (19\%), stable disease (SD) in 17 patients (28\%), and progressive disease (PD) in 33 patients $(55 \%)$. Thirteen patients (21\%) met the HPD criteria. Among HPD patients, $\mathrm{TGK}_{\mathrm{R}}$ ranged from 2.0 to 25.9. No progressive disease followed by tumor shrinkage (i.e., pseudoprogression) was observed. The changes in the sum of the largest diameters of target lesions according to response classification are shown in Fig. 2.

The median PFS according to RECIST v1.1 was 2.0 (95\% CI, 1.5-2.5) months, and the median OS was not reached. Patients who showed HPD had a significantly shorter PFS (median: 0.7 months vs. 2.4 months, $P<0.001$, HR 4.8) (Fig. 3a), and OS (median: 2.3 months vs. not reached, $P<0.001$, HR 9.2) than those of the patients who did not show HPD (Fig. 3b). 


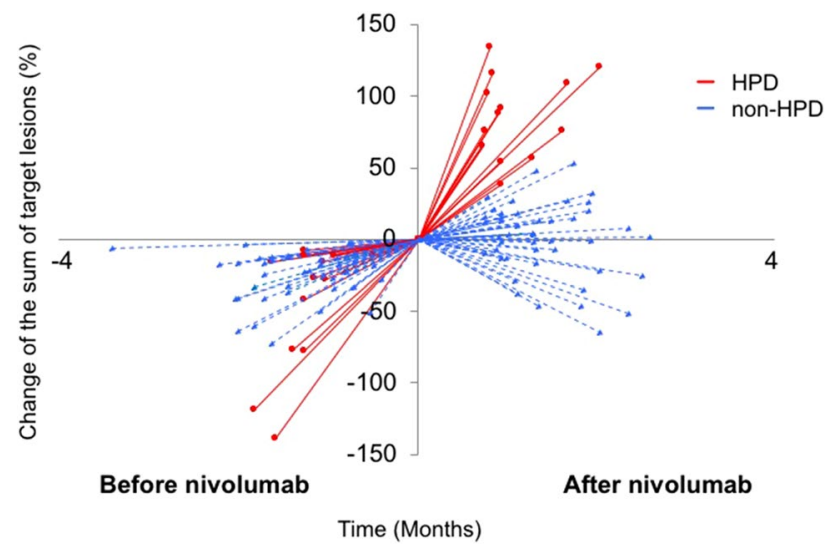

Fig. 2 Spider plot depicting percentage change in the sum of the largest diameters of target lesions over time according to hyperprogressive disease (HPD) status

\section{Clinicopathological and molecular features associated with HPD}

Among baseline clinical characteristics, the frequencies of an ECOG PS of 1 or 2 (77\% vs. $29 \%, P=0.003)$ and liver metastases ( $77 \%$ vs. $41 \%, P=0.029$ ) were significantly higher in the HPD group than in the non-HPD group (Table 1). Furthermore, the sum of diameters of target lesions at baseline was significantly larger in the HPD group (median $104.2 \mathrm{~mm}$ vs. $44.9 \mathrm{~mm}, P=0.003$ ) than in the non-HPD group (Table 1). While nine of the 13 (69\%) patients who showed HPD presented with all of these clinical factors-an ECOG PS of 1 or 2, liver metastases, and sum of diameters larger than or equal to the medianonly one patient who did not present these factors showed
HPD (Additional File 1: Table S2). There were no other clinical factors significantly associated with HPD. OS was worse with HPD than non-HPD even after adjusting other clinical factors including ECOG PS, liver metastasis, and sum of diameters $(P<0.001$, HR 6.1) (Additional File 1: Table S3).

There were no significant differences in HER2, PD-L1, MMR, and EBV statuses between the two groups (Table 2). Each one of the patients with the D-MMR and positive EBV experienced HPD. One patient with the D-MMR had an ECOG PS of 2, liver and skin metastases, $S_{0}$ larger than the median, and somatic mutations in multiple genes, including $F B X W 7$ and $P T E N$. Another patient positive for EBV had $F B X W 7$ mutation and $M Y C$ amplification.

In the HPD group, three patients showed KRAS amplification, while three others showed $F B X W 7$ mutation; these findings were not observed in the non-HPD group $(P=0.009)$. Mutations in CDKN2A and PTEN and FGF 19 amplification were also observed in only the HPD group; however, there were no statistically significant differences between the HPD and the non-HPD groups (Table 2).

With regard to laboratory data, baseline ANC (median: 4,490/ 1 vs. $2,720 / \mu 1, P=0.002$ ), CRP level (median: $4.0 \mathrm{mg} / \mathrm{dl}$ vs. $0.50 \mathrm{mg} / \mathrm{dl}, P=0.006)$, and LDH level (median: $396.0 \mathrm{U} / 1$ vs. $179.5 \mathrm{U} / 1, P=0.006$ ) were significantly higher in the HPD group than in the non-HPD group (Table 3). Furthermore, analyses of serial laboratory data showed that ANC and CRP levels were significantly elevated at 4 weeks after the initiation of nivolumab treatment (ANC, $4490 / \mu \mathrm{l}$ vs. $7740 / \mu \mathrm{l}$; CRP, $4.0 \mathrm{mg} / \mathrm{dl}$ vs. $8.3 \mathrm{mg} /$ dl) in the HPD group (Fig. 4). On the other hand, in the non-HPD group, there were no significant differences in laboratory data during treatment courses.
Fig. 3 Progression-free survival (PFS) a and overall survival (OS) b according to hyperprogressive disease (HPD) status

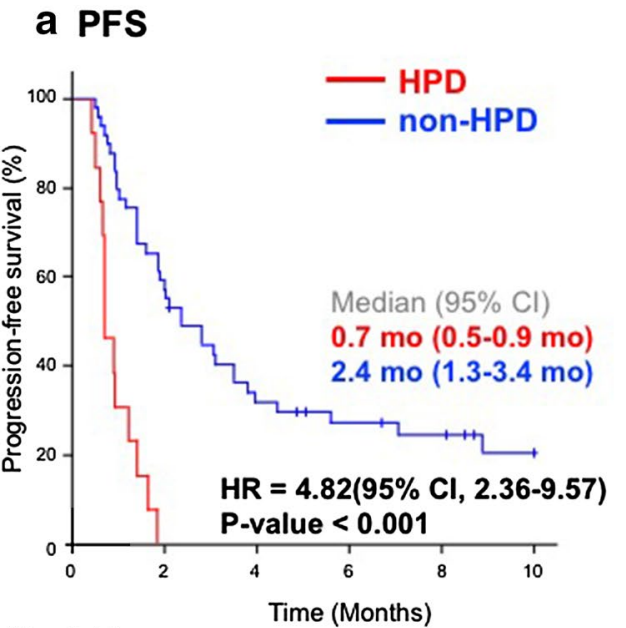

No. at risk

$\begin{array}{cccccc}13 & 0 & 0 & 0 & 0 & 0 \\ 49 & 29 & 15 & 11 & 9 & 5\end{array}$

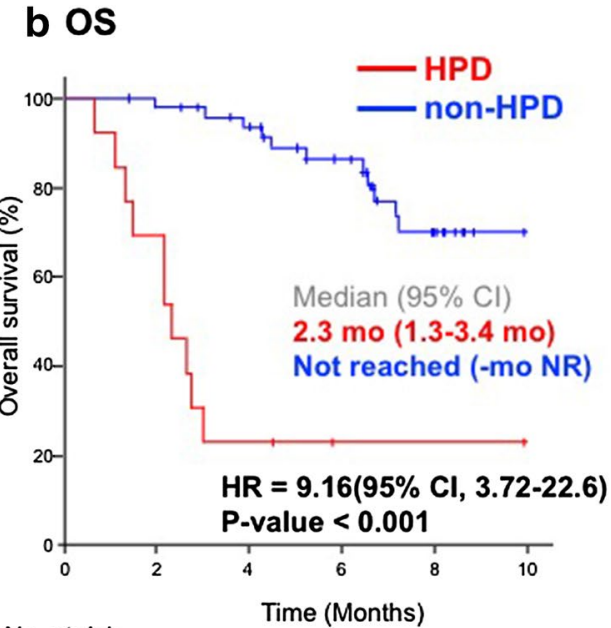

No. at risk

$\begin{array}{cccccc}13 & 9 & 3 & 1 & 1 & 1 \\ 49 & 47 & 44 & 33 & 22 & 10\end{array}$


Table 2 Patients' characteristics (molecular profiles)

\begin{tabular}{|c|c|c|c|c|c|c|}
\hline Features & Available & Non-HPD $(n=49)$ & $\operatorname{HPD}(n=13)$ & HPD ratio $(\%)$ & OR & $P$ value \\
\hline HER2, $n(\%)$ & 61 & & & & & \\
\hline Negative & & $36(75.0)$ & $11(84.6)$ & 23.4 & Ref. & 0.71 \\
\hline Positive & & $12(25.0)$ & $2(15.4)$ & 14.3 & 0.56 & \\
\hline MMR, $n(\%)$ & 55 & & & & & \\
\hline Proficient & & $36(83.7)$ & $11(91.7)$ & 23.4 & Ref. & 0.66 \\
\hline Deficient & & $7(16.3)$ & $1(8.3)$ & 12.5 & 0.48 & \\
\hline $\mathrm{EBV}, n(\%)$ & 57 & & & & & \\
\hline Negative & & $41(93.2)$ & $12(92.3)$ & 22.6 & Ref. & 1.00 \\
\hline Positive & & $3(6.8)$ & $1(7.7)$ & 25 & 1.1 & \\
\hline PD-L1, $n(\%)$ & 53 & & & & & \\
\hline Positive in $\mathrm{TC}$ & & $11(26.8)$ & $1(8.3)$ & 8.3 & 0.25 & 0.26 \\
\hline Positive in IC & & $36(87.8)$ & $11(91.7)$ & 23.4 & 1.5 & 1.00 \\
\hline Any expression & & $36(87.8)$ & $11(91.7)$ & 23.4 & Ref. & 1.00 \\
\hline No expression & & $5(12.2)$ & $1(8.3)$ & 16.7 & 0.67 & \\
\hline PD-L1 CPS, $n(\%)$ & 53 & & & & & \\
\hline$<10$ & & $30(73.2)$ & $9(75.0)$ & 23.1 & Ref. & 1.00 \\
\hline$\geq 10$ & & $11(26.8)$ & $3(25.0)$ & 21.4 & 0.91 & \\
\hline $\mathrm{TMB}, n(\%)$ & 47 & & & & & \\
\hline$<10$ & & $16(43.2)$ & $1(10.0)$ & 5.9 & Ref. & 0.067 \\
\hline$\geq 10$ & & $21(56.8)$ & $9(90.0)$ & 30 & 7.3 & \\
\hline Genomic alterations & 47 & & & & & \\
\hline$C C N D 1$ amplification & & 1 & 0 & 0 & 0.83 & 1.00 \\
\hline CCNE1 amplification & & 3 & 0 & 0 & 0.33 & 0.55 \\
\hline$C D K N 2 A$ mutation & & 0 & 1 & 100 & 9.3 & 0.28 \\
\hline$E R B B 2$ mutation & & 1 & 1 & 50 & 2.8 & 0.48 \\
\hline$E R B B 2$ amplification & & 6 & 2 & 25 & 0.85 & 1.00 \\
\hline FGF19 amplification & & 0 & 1 & 100 & 9.3 & 0.28 \\
\hline$F G F R 2$ amplification & & 1 & 0 & 0 & 0.83 & 1.00 \\
\hline$F B X W 7$ mutation & & 0 & 3 & 100 & 26.2 & 0.018 \\
\hline$K R A S$ mutation & & 2 & 1 & 33.3 & 1.3 & 1.00 \\
\hline$K R A S$ amplification & & 0 & 3 & 100 & 26.2 & 0.018 \\
\hline$M D M 2$ amplification & & 1 & 1 & 50 & 2.8 & 0.48 \\
\hline$M Y C$ amplification & & 1 & 1 & 50 & 2.8 & 0.48 \\
\hline NF1 mutation & & 1 & 0 & 0 & 0.83 & 1.00 \\
\hline$P I K 3 C A$ mutation & & 4 & 3 & 42.9 & 2.3 & 0.38 \\
\hline PTEN mutation & & 0 & 1 & 100 & 9.3 & 0.28 \\
\hline RHOA mutation & & 1 & 1 & 50 & 2.8 & 0.48 \\
\hline TP53 mutation & & 17 & 7 & 29.2 & 1.2 & 1.00 \\
\hline
\end{tabular}

$H E R 2$ human epidermal growth factor receptor related 2, $M M R$ mismatch repair $E B V$ Epstein-Barr virus, $P D-L 1$ programmed death-ligand 1, TC tumor cell, $C P S$ combined positive score, $T M B$ tumor mutation burden

\section{Discussion}

We assessed HPD by tumor growth kinetics in patients with AGC who received nivolumab treatment, and evaluated the clinicopathological and molecular factors associated with HPD. In this study, $22 \%$ of patients with AGC showed HPD during nivolumab treatment and several factors were associated with HPD. To the best of our knowledge, this is the first study to report the characteristics of
HPD in AGC patients. Previous studies reported that HPD was observed in 9-29\% of various types of cancers treated with anti-PD-1/PD-L1 mAbs $[15,16]$; this finding is in agreement with the results of our study. In the ATTRAC TION-2 trial, no significant difference of the frequency of patients with early tumor progression was reported between nivolumab and placebo group [21]. However, since they did not assess the tumor growth rate in the previous treatment, which is needed to determine HPD status, 
Table 3 Patients' characteristics (baseline laboratory data)

\begin{tabular}{lccll}
\hline Features & Non-HPD $(n=49)$ & HPD $(n=13)$ & $\begin{array}{l}\text { OR }(\geq \text { median } \\
\text { vs. }<\text { median })\end{array}$ & $P$ value \\
\hline CEA $(\mathrm{ng} / \mathrm{ml})$, range & $17.7(1.0-1789.0)$ & $5.7(4.0-415.2)$ & 0.65 & 0.74 \\
CA19-9 $(\mathrm{U} / \mathrm{ml})$, range & $26.6(5.3-4158.0)$ & $294.0(0.6-5545.0)$ & 1.5 & 0.63 \\
ANC $\left(\mathrm{x} 10^{2} / \mu \mathrm{l}\right)$, range & $27.2(8.3-100.6)$ & $44.9(31.1-103.8)$ & 8.0 & 0.002 \\
Lymphocyte $\left(\mathrm{x} 10^{2} / \mu \mathrm{l}\right)$, range & $12.9(4.1-19.0)$ & $10.4(4.5-18.5)$ & 0.82 & 0.60 \\
NLR, range & $2.2(0.7-24.5)$ & $4.9(2.4-11.3)$ & 3.0 & 0.008 \\
CRP $(\mathrm{mg} / \mathrm{dl})$, range & $0.5(0.05-10.3)$ & $4.0(0.4-8.7)$ & 4.1 & 0.006 \\
LDH $(\mathrm{U} / \mathrm{l})$, range & $179.5(121.0-524.0)$ & $396.0(182.0-2392.0)$ & 5.3 & 0.006 \\
Albumin $(\mathrm{g} / \mathrm{dl})$, range & $3.4(2.5-4.2)$ & $3.1(2.1-4.2)$ & 0.56 & 0.24 \\
\hline
\end{tabular}

$H P D$ hyperprogressive disease, $O R$ odds ratio, $A N C$ absolute neutrophil count, $N L R$ neutrophil-to-lymphocyte ratio, $C R P C$-reactive protein, $L D H$ lactate dehydrogenase
Fig. 4 Transition of absolute neutrophil count (ANC) (a) and CRP (b) after nivolumab

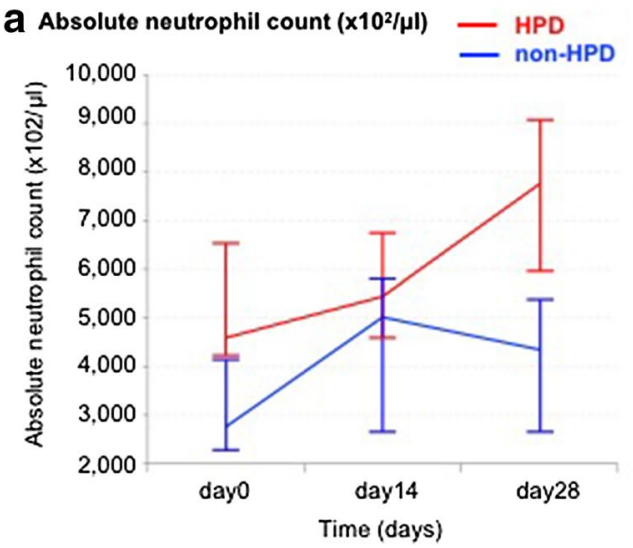

it was not evaluated whether tumor increase was accelerated or not in each group.

In this study, survival outcome of patients with HPD was shorter than those with non-HPD as previously reported in other cancer type, though the impact of HPD on the survival was not clear because of the lack of control. Actually, OS of patients with PD treated with nivolumab was not worse than those with placebo in the ATTRACTION-2 [22]. However, exact OS after HPD remains unclear; thus, further analysis might be necessary.

Although the mechanism of HPD is unclear, upregulation of alternative immune checkpoints resulting in further immune suppression [23], acceleration of cell growth by blocking of cancer intrinsic PD-1 [24], low number of senescent CD4 T cells, and FcR triggering of clustered macrophages with a specific immunophenotyped [25] have been suggested to cause rapid disease progression by PD1 blockade [17]. Most recently, we reported an increase in regulatory $\mathrm{T}$ cells with proliferative capacity among tumor-infiltrating lymphocytes in AGC patients who showed HPD after treatment with an anti-PD-1 mAb [26]. Furthermore, an in vitro study showed that PD-1 blockade activated not only effector $\mathrm{T}$ cells, but also Treg cells, which promoted tumor progression in a fraction of patients [26].

In the previous studies, only higher age and regional recurrence in head and neck cancer were suggested as clinical factors associated with HPD [15, 16, 18]. However, we found that AGC patients with poor PS, liver metastasis, or a large tumor at baseline more commonly experienced hyperprogression after nivolumab treatment compared to those without these factors. Several studies suggested an association between resistance to immune checkpoint inhibitors and low baseline PS in various types of cancers, including gastric cancer [7, 9, 13, 14, 27]. Severe exacerbations of primary diseases in NSCLC patients with poor PS were also reported in a case series [28]. Although the exact cause of worse outcome in patients with poor PS remains unclear, patients with poor PS may not stay on treatment long enough to achieve a response. Another study suggested higher clearance of anti-PD1 therapy in patients with cancer cachexia and catabolic clearance to be a cause of HPD; however, it may not be sufficient to induce HPD [29].

Liver metastasis has also been suggested to decrease the probability of a response to anti-PD-1/PD-L1 mAbs by liver-induced immune tolerance [30-33]. Tumor burden was 
shown to negatively affect tumor response and survival after anti-PD-1 mAb treatment, especially when T-cell reinvigoration in peripheral blood was not sufficient $[34,35]$. Our findings indicated that these factors could be associated with not only resistance to anti-PD-1 therapy, but also rapid tumor progression caused by inhibition of PD-1 in AGC.

Although all patients with KRAS amplification or FBXW7 mutation showed HPD in our study, the association between these genetic alterations and HPD was not clear. These alterations could be only prognostic factors. Therefore, a validation in a larger cohort with nivolumab and other treatments are needed. A previous study regarding genomic analyses of patients with HPD during treatment with immune checkpoint inhibitors suggested the association between MDM2 amplification and HPD, but one of the two patients with $M D M 2$ amplification did not show HPD in our study.

Although a D-MMR status and EBV positivity were suggested to be associated with better responses to antiPD-1/PD-L1 mAbs in previous case series [36, 37], each all patients with the D-MMR status or EBV positivity showed HPD in our cohort. The patient with the D-MMR had some HPD-associated features, such as an ECOG PS of 2, liver metastases, and a large tumor size. In addition, both patients received nivolumab as fourth line treatment, which suggested that late initiation of nivolumab treatment could affect the poor outcome in these patients. These findings suggest that even patients with favorable molecular characteristics for anti-PD-1/PD-L1 therapy can have HPD, especially when having HPD-associated factors.

We also found that ANC and CRP levels significantly increased during nivolumab treatment in patients with HPD. Increased ANC generally reflects a premature release of myeloid cells from the bone marrow. Recent evidence suggests that accumulation of myeloid-derived suppressor cells (MDSCs) is related to resistance to immune checkpoint inhibitors [38]. MDSC counts were also reported to be positively correlated with CRP levels [39]. Although we did not assess the MDSC fraction in neutrophils, the increased ANC and CRP level could be due to the increase in the MDSC fraction, which potentially reflected hyperprogressive status in our study. Changes in these laboratory data can be good on-treatment markers for early HPD prediction, but further investigations of these immune phenotypes are needed.

It is important to note the limitations of the present study. First, this was a retrospective and single-institution study with a limited sample size. Therefore, the differences of clinicopathological characteristics between HPD and nonHPD group contribute to poor PFS and OS by HPD. Second, since we did not evaluate TGKs of patients treated with nivolumab in comparison with those of patients treated with chemotherapy or supportive care alone, we could not accurately assess whether HPD in our patient population was specifically caused by nivolumab or was a natural course.
Prospective observational study is necessary to compare treatment course after each treatment. Third, we used RECIST v1.1 for assessing tumor burden, because of which a limited number of tumors were considered in each organ. Thus, the total tumor burden was potentially underestimated. Moreover, we could not evaluate non-target lesions, including ascites, pleural effusion, and bone metastases. Finally, we applied the definition of HPD used in the previous studies [15-17], which has not been standardized. Although the ordinary definition of HPD has been based on a hypothesis that tumor growth rate is stable over time without treatment, it has been suggested to be affected by several factors, including histologic subtypes and tumor size [40]. Because of all these limitations, the current study is only hypothesisgenerating, and mechanisms and biomarkers of HPD in AGC treated with nivolumab need to be further evaluated.

\section{Conclusions}

Although the small sample size is the major limitation of our study, our study suggested that some clinicopathological and molecular characteristics might be associated with HPD in AGC patients treated with nivolumab. The identification of these characteristics will potentially allow careful management of AGC during nivolumab treatment. However, further investigations in larger cohorts are needed to confirm HPDassociated biomarkers.

Author contributions AS, YN, SM, AK, and KS designed the study, collected data, performed data analysis, and wrote manuscript. YK, $\mathrm{HB}, \mathrm{TD}, \mathrm{TY}, \mathrm{TK}$, and TA were involved in data interpretation and critically reviewing the manuscript. TK was involved in testing tumor tissue as well as critically reviewing the manuscript. All authors read and approved the final manuscript.

Funding This study was supported by a research funding from National Cancer Center Hospital East (none apply).

Data Availability All data analyzed during this study have been included within the article.

\section{Compliance with ethical standards}

Ethics approval and consent to participate All procedures followed in this study were in accordance with the Declaration of Helsinki of 1964 and later versions and the Japanese Ethical Guidelines for Medical and Health Research Involving Human Subjects. Informed consent for it was obtained from all patients for their being included in the study.

Consent for publication This is not applicable for this study.

Conflict of interest AS has nothing to disclose. YN reports personal fees from Chugai. SM has nothing to disclose. AK reports research funding from Ono, Sumitomo Dainippon, and Taiho. YK reports consulting or advisory role for Takeda; personal fees from Bayer, Lilly, 
and Taiho; and research funding from Astra Zeneca, Daiichi Sankyo, Incyte, Taiho, and Takeda. HB reports research funding from Astra Zeneca and Sysmex. TK reports personal fees from MSD; and research funding from Astellas, Bristol-Myers Squibb, MSD, Oncolys BioPharma, Ono, Shionogi. TD reports consulting or advisory role for Amgen, Chugai, Daiichi Sankyo, Kyowa Hakko Kirin, Lilly, MSD, Sumitomo Dainippon, and Taiho; and research funding from Abbvie, Astellas, Boehringer Ingelheim, Bristol-Myers Squibb, Celgene, Chugai, Daiichi Sankyo, Janssen, Kyowa Hakko Kirin, Lilly, Merck Serono, MSD, Novartis, Pfizer, Quintiles, Sumitomo Group, Takeda, and Taiho. AO reports personal fees from Bristol-Myers Squibb, Chugai, Ono, and Taiho. TY consulting or advisory role for Chugai, Lilly, Merck Serono, and Sanofi; and research funding from Chugai, GlaxoSmithKline, MSD, Nippon Boehringer Ingelheim, Sanofi, and Sumitomo Dainippon. TK consulting or advisory role for ThermoFisher Inc; personal fees from Chugai and Roche Diagnostics; and research funding from Daiichi Sankyo. TA has nothing to disclose. KS reports consulting or advisory role for Astellas, Bristol-Myers Squibb, Lilly, Ono, Pfizer, and Takeda; personal fees from AbbVie, Novartis, and Yakult; and research funding from Chugai, Daiichi Sankyo, Lilly, MSD, Ono, Sumitomo Dainippon, and Taiho.

\section{References}

1. Ferlay J, Soerjomataram I, Dikshit R, Eser S, Mathers C, Rebelo $\mathrm{M}$, et al. Cancer incidence and mortality worldwide: sources, methods and major patterns in GLOBOCAN 2012. Int J Cancer. 2015;136(5):E359-86.

2. Koizumi W, Narahara H, Hara T, Takagane A, Akiya T, Takagi M, et al. S-1 plus cisplatin versus S-1 alone for first-line treatment of advanced gastric cancer (SPIRITS trial): a phase III trial. Lancet Oncol. 2008;9(3):215-21.

3. Cunningham D, Starling N, Rao S, Iveson T, Nicolson M, Coxon $\mathrm{F}$, et al. Capecitabine and oxaliplatin for advanced esophagogastric cancer. N Engl J Med. 2008;358(1):36-46.

4. Wilke H, Muro K, Van Cutsem E, Oh SC, Bodoky G, Shimada $\mathrm{Y}$, et al. Ramucirumab plus paclitaxel versus placebo plus paclitaxel in patients with previously treated advanced gastric or gastro-oesophageal junction adenocarcinoma (RAINBOW): a double-blind, randomised phase 3 trial. Lancet Oncol. 2014;15(11):1224-35.

5. Janowitz T, Thuss-Patience P, Marshall A, Kang JH, Connell $\mathrm{C}$, Cook N, et al. Chemotherapy vs supportive care alone for relapsed gastric, gastroesophageal junction, and oesophageal adenocarcinoma: a meta-analysis of patient-level data. Br J Cancer. 2016;114(4):381-7.

6. Fuchs CS, Tomasek J, Yong CJ, Dumitru F, Passalacqua R, Goswami C, et al. Ramucirumab monotherapy for previously treated advanced gastric or gastro-oesophageal junction adenocarcinoma (REGARD): an international, randomised, multicentre, placebocontrolled, phase 3 trial. Lancet. 2014;383(9911):31-9.

7. Robert C, Long GV, Brady B, Dutriaux C, Maio M, Mortier L, et al. Nivolumab in previously untreated melanoma without BRAF mutation. N Engl J Med. 2015;372(4):320-30.

8. Borghaei H, Paz-Ares L, Horn L, Spigel DR, Steins M, Ready $\mathrm{NE}$, et al. Nivolumab versus docetaxel in advanced nonsquamous non-small-cell lung cancer. N Engl J Med. 2015;373(17):1627-39.

9. Motzer RJ, Escudier B, McDermott DF, George S, Hammers HJ, Srinivas $\mathrm{S}$, et al. Nivolumab versus everolimus in advanced renalcell carcinoma. N Engl J Med. 2015;373(19):1803-13.

10. Younes A, Santoro A, Shipp M, Zinzani PL, Timmerman JM, Ansell S, et al. Nivolumab for classical Hodgkin's lymphoma after failure of both autologous stem-cell transplantation and brentuximab vedotin: a multicentre, multicohort, single-arm phase 2 trial. Lancet Oncol. 2016;17(9):1283-94.

11. Ferris RL, Blumenschein G Jr, Fayette J, Guigay J, Colevas AD, Licitra L, et al. Nivolumab for recurrent squamous-cell carcinoma of the head and neck. N Engl J Med. 2016;375(19):1856-67.

12. Kang YK, Boku N, Satoh T, Ryu MH, Chao Y, Kato K, et al. Nivolumab in patients with advanced gastric or gastro-oesophageal junction cancer refractory to, or intolerant of, at least two previous chemotherapy regimens (ONO-4538-12, ATTRAC TION-2): a randomised, double-blind, placebo-controlled, phase 3 trial. Lancet. 2017;390(10111):2461-71.

13. Fuchs CS, Doi T, Jang RW, Muro K, Satoh T, Machado M, et al. Safety and efficacy of pembrolizumab monotherapy in patients with previously treated advanced gastric and gastroesophageal junction cancer: phase 2 clinical KEYNOTE-059 trial. JAMA Oncol. 2018;4(5):e180013.

14. Shitara K, Ozguroglu M, Bang YJ, Bartolomeo MD, Mandala M, Ryu MH, et al. Pembrolizumab versus paclitaxel for previously treated, advanced gastric or gastro-oesophageal junction cancer (KEYNOTE-061): a randomised, open-label, controlled, phase 3 trial. Lancet. 2018;392(10142):123-33.

15. Champiat S, Dercle L, Ammari S, Massard C, Hollebecque A, Postel-Vinay $\mathrm{S}$, et al. Hyperprogressive disease is a new pattern of progression in cancer patients treated by Anti-PD-1/PD-L1. Clin Cancer Res. 2017;23(8):1920-8.

16. Saada-Bouzid E, Defaucheux C, Karabajakian A, Coloma VP, Servois V, Paoletti X, et al. Hyperprogression during antiPD-1/PD-L1 therapy in patients with recurrent and/or metastatic head and neck squamous cell carcinoma. Ann Oncol. 2017;28(7):1605-11.

17. Kato S, Goodman A, Walavalkar V, Barkauskas DA, Sharabi A, Kurzrock R. Hyperprogressors after immunotherapy: analysis of genomic alterations associated with accelerated growth rate. Clin Cancer Res. 2017;23(15):4242-50.

18. Kurman JS, Murgu SD. Hyperprogressive disease in patients with non-small cell lung cancer on immunotherapy. J Thorac Dis. 2018;10(2):1124-8.

19. Eisenhauer EA, Therasse P, Bogaerts J, Schwartz LH, Sargent D, Ford R, et al. New response evaluation criteria in solid tumours: revised RECIST guideline (version 1.1). Eur J Cancer. 2009;45(2):228-47.

20. Kawazoe A, Shitara K, Kuboki Y, Bando H, Kojima T, Yoshino T, et al. Clinicopathological features of 22C3 PD-L1 expression with mismatch repair, Epstein-Barr virus status, and cancer genome alterations in metastatic gastric cancer. Gastric Cancer. 2018. https ://doi.org/10.1007/S10120-018-0843-9.

21. Feng YN, Zwirtes P, Reshef R, Plautz D, Boku G, Chen N, et al Roy A, Sheng J Evaluating the occurrence of early tumor progression (ETP) in patients with gastric cancer treated with nivolumab versus placebo. In: Paper presented at the proceedings of the society for immunotherapy of cancer (SITC) 33rd annual meeting, 2018. pp. 833-4.

22. Satoh TC, Kang L, Chao Y, Kato Y, Chung K, Chen H, et al. A phase III study of nivolumab (nivo) in previously treated advanced gastric or gastric esophageal junction (G/GEJ) cancer (ATTRACTION-2): two-years update data. Ann Oncol. 2018;29(suppl_8):viii205-70.

23. Koyama S, Akbay EA, Li YY, Herter-Sprie GS, Buczkowski KA, Richards WG, et al. Adaptive resistance to therapeutic PD-1 blockade is associated with upregulation of alternative immune checkpoints. Nat Commun. 2016;7:10501.

24. Du S, McCall N, Park K, Guan Q, Fontina P, Ertel A, et al. Blockade of tumor-expressed PD-1 promotes lung cancer growth. Oncoimmunology. 2018;7(4):e1408747.

25. Lo Russo G, Moro M, Sommariva M, Cancila V, Boeri M, Centonze G, et al. Antibody-Fc/FcR interaction on macrophages as 
a mechanism for hyperprogressive disease in non-small cell lung cancer subsequent to PD-1/PD-L1 blockade. Clin Cancer Res. 2018. https://doi.org/10.1158/1078-0432.CCR-18-1390.

26. Togashi Y, Sasaki KTA, et al. Clinicopathological, genomic and immunological features of hyperprogressive disease during PD-1 blockade in gastric cancer patients. J Clin Oncol. 2018. https://doi. org/10.1200/JCO.2018.36.15_suppl.4106.

27. Carbone DP, Reck M, Paz-Ares L, Creelan B, Horn L, Steins M, et al. First-line nivolumab in stage IV or recurrent non-small-cell lung cancer. N Engl J Med. 2017;376(25):2415-26.

28. Kanai O, Fujita K, Okamura M, Nakatani K, Mio T. Severe exacerbation or manifestation of primary disease related to nivolumab in non-small-cell lung cancer patients with poor performance status or brain metastases. Ann Oncol. 2016;27(7):1354-6.

29. Turner D, Kondic AG, Anderson KM, Robinson A, Garon EB, Riess JW, et al. Pembrolizumab exposure-response assessments challenged by association of cancer cachexia and catabolic clearance. Clin Cancer Res. 2018;24:5841-9.

30. Bearz A, Perin T, Cancian L, Berto E, Sartor I, Tirelli U. Immune checkpoint inhibitors and response analysis: a tough challenge. A case report. BMC Res Notes. 2016;9:349.

31. Massard C, Gordon MS, Sharma S, Rafii S, Wainberg ZA, Luke J, et al. Safety and efficacy of durvalumab (MEDI4736), an antiprogrammed cell death ligand-1 immune checkpoint inhibitor, in patients with advanced urothelial bladder cancer. J Clin Oncol. 2016;34(26):3119-25.

32. Tumeh PC, Hellmann MD, Hamid O, Tsai KK, Loo KL, Gubens MA, et al. Liver metastasis and treatment outcome with AntiPD-1 monoclonal antibody in patients with melanoma and NSCLC. Cancer Immunol Res. 2017;5(5):417-24.

33. Funazo T, Nomizo T, Kim YH. Liver metastasis is associated with poor progression-free survival in patients with non-small cell lung cancer treated with nivolumab. J Thorac Oncol. 2017;12(9):e140-1.
34. Huang AC, Postow MA, Orlowski RJ, Mick R, Bengsch B, Manne $\mathrm{S}$, et al. T-cell invigoration to tumour burden ratio associated with anti-PD-1 response. Nature. 2017;545(7652):60-5.

35. Joseph RW, Elassaiss-Schaap J, Kefford R, Hwu WJ, Wolchok JD, Joshua AM, et al. Baseline tumor size is an independent prognostic factor for overall survival in patients with melanoma treated with pembrolizumab. Clin Cancer Res. 2018;24:4960-7.

36. Kim ST, Cristescu R, Bass AJ, Kim KM, Odegaard JI, Kim $\mathrm{K}$, et al. Comprehensive molecular characterization of clinical responses to PD-1 inhibition in metastatic gastric cancer. Nat Med. 2018;24:1449.

37. Janjigian YY, Sanchez-Vega F, Jonsson P, Chatila WK, Hechtman $\mathrm{JF}, \mathrm{Ku} \mathrm{GY}$, et al. Genetic predictors of response to systemic therapy in esophagogastric cancer. Cancer Discov. 2018;8(1):49-58.

38. Engblom C, Pfirschke C, Pittet MJ. The role of myeloid cells in cancer therapies. Nat Rev Cancer. 2016;16(7):447-62.

39. Gonda K, Shibata M, Ohtake T, Matsumoto Y, Tachibana K, Abe $\mathrm{N}$, et al. Myeloid-derived suppressor cells are increased and correlated with type 2 immune responses, malnutrition, inflammation, and poor prognosis in patients with breast cancer. Oncol Lett. 2017;14(2):1766-74.

40. Le Tourneau C, Paoletti X, Coquan E, Sablin MP, Zoubir M, Tannock IF. Critical evaluation of disease stabilization as a measure of activity of systemic therapy: lessons from trials with arms in which patients do not receive active treatment. J Clin Oncol. 2014;32(3):260-3.

Publisher's Note Springer Nature remains neutral with regard to jurisdictional claims in published maps and institutional affiliations.

\section{Affiliations}

\section{Akinori Sasaki $^{1,2}$ - Yoshiaki Nakamura ${ }^{1}$ Saori Mishima ${ }^{1}$. Akihito Kawazoe ${ }^{1}$ - Yasutoshi Kuboki ${ }^{1} \cdot$ Hideaki Bando $^{1}$. Takashi Kojima ${ }^{1} \cdot$ Toshihiko Doi $^{1}$ - Atsushi Ohtsu ${ }^{1} \cdot$ Takayuki Yoshino $^{1} \cdot$ Takeshi Kuwata $^{3} \cdot$ Tetsuo Akimoto $^{2,4}$. Kohei Shitara'}

\author{
Akinori Sasaki \\ akisasak@east.ncc.go.jp \\ Yoshiaki Nakamura \\ yoshinak@east.ncc.go.jp \\ Saori Mishima \\ smishima@east.ncc.go.jp \\ Akihito Kawazoe \\ akawazoe@east.ncc.go.jp \\ Yasutoshi Kuboki \\ ykuboki@east.ncc.go.jp \\ Hideaki Bando \\ hbando@aichi-cc.jp \\ Takashi Kojima \\ takojima@east.ncc.go.jp \\ Toshihiko Doi \\ tdoi@east.ncc.go.jp \\ Atsushi Ohtsu \\ aohtsu@east.ncc.go.jp
}

Takayuki Yoshino

tyoshino@east.ncc.go.jp

Takeshi Kuwata

tkuwata@east.ncc.go.jp

Tetsuo Akimoto

takimoto@east.ncc.go.jp

1 Department of Gastroenterology and Gastrointestinal Oncology, National Cancer Center Hospital East, 6-5-1 Kashiwanoha, Kashiwa, Chiba 277-8577, Japan

2 Courses of Advanced Clinical Research of Cancer, Juntendo University Graduate School of Medicine, Tokyo, Japan

3 Department of Pathology and Clinical Laboratories, National Cancer Center Hospital East, Kashiwa, Chiba, Japan

4 Department of Radiation Oncology, National Cancer Center Hospital East, Kashiwa, Chiba, Japan 